\title{
Analysis of Surface Flashover of $33 k V$ Pin Type Insulators due to Saline Pollution
}

\author{
N.H.C Janaka, J.R Lucas and D.G.R Fernando
}

\begin{abstract}
Contamination-driven insulator failure is a problem that incessantly plagues distribution systems. Contamination levels can continue to grow unless abated by natural cleaning or if not taken measures to wash insulators at the distribution level in a preventative maintenance mode. In order to assess the pollution behavior, $33 \mathrm{kV}$ pin type insulator was selected as a sample insulator and been subjected for natural pollution at three selected localities (zone 1,2 and 3) for considerable period. The naturally polluted insulators have been subjected for conductivity test and by which the equivalent salt deposit density (ESDD) is calculated. Subsequently, artificially contaminated insulators of different pollution severities were tested for power frequency and impulse test in the High Voltage Laboratory of University of Moratuwa. Finally, it is recommended that insulators in Zone 1 have to be treated after 8 months from the date of last treatment and those in Zone $2 \& 3$ to be treated after 18 months under preventive maintenance to get away from flashover. It is also recommended to review the required specific creepage distance (SCD) of insulators installed in non-polluted areas due to the fact that the current practice of insulators placement in most of the places are with SCD of $25 \mathrm{~mm} / \mathrm{kV}$ which is recommended for high polluted zones as per IEC regulation.
\end{abstract}

Keywords: Insulator, Surface flashover, Equivalent Salt Deposit Density (ESDD), surface conductivity, pollution severity, specific creepage distance.

\section{Introduction}

The efficacy of the power system is based mainly on the continuity of the service; avoidance of faults that cause economical losses for the utility and the user. To maintain this continuity, one of the main problems is the effect produced by pollution in the overhead line insulators. Pollution is one of the main causes of flashover in insulators. Pollution can be caused through a variety of sources, (such as sea salt spray, industrial pollution, ash). The wind is the main bearer of the pollution particles, with the gravity and the electric field having a secondary role. By the action of rain, fog, etc. the pollution layer on the surface is dampened and increases the conductivity. Although many factors can be used to define the pollution of insulators, three types of pollution are usually categorised: industrial, saline and desert. Insulator flashover due to saline pollution has gained special importance in Sri Lanka in recent years, especialy in the operation in coastal areas.

\section{Objective}

The main objective of the paper is to predict the flashover voltage of the $33 \mathrm{kV}$ insulators due to saline pollution and to streamline the preventive maintenance procedure to treat the polluted insulators and further to review the specific creepage distance of insulators based on polluted zones and co-relate the same with actual scenarios.

\section{Scope of work}

The scope of the work is as given below;

- Data collection of insulator flashover in Hambantota and Tissamaharama areas over one year period.

- Conductivity test on polluted insulators

- Collection of Rainfall and Thunder Day data in the region selected (Hambantota) over the last three year period.

- Streamline preventive maintenance procedure

- Prediction of flashover voltages (FOV's) with Equivalent salt deposit density (ESDD) and analyze the results.

- Co-relate the results with the IEC regulations to select the specific creepage distance of the insulators.

Eng. N.H.C. Janaka, B.Sc. Eng. (Ruhuna), M.Sc. (Moratuwa), AMIE(Sri Lanka), Electrical Engineer, Ceylon Electricity Board.

Eng. (Prof.) J.R. Lucas, B.Sc. Eng.(Cey), C.Eng., M.Sc.(Manch), PhD.(Manch), FIEE, FIE (Sri Lanka), IPEng, MCS (Sri Lanka), Senior Professor of Electrical Engineering, University of Moratuwa.

Eng. D.G. Rienzie Fernando, B.Sc. Eng.(Moratuwa), C.Eng., MIE(Sri Lanka), Managing Director, Amithi Power Consultant (Pvt) Ltd. 


\section{Analysis of Insulator flashover}

A survey has been carried out in Hambantota and Tissamaharama areas where they are very much closer to the coastal belt and to check the status of system breakdowns due to $33 \mathrm{kV}$ insulator flashover. Key findings of the survey have been tabulated over the year 2009. Detail data is available in Table 1 below.

Table-1 Insulator flashover records in year 2009 (April-Sep.)

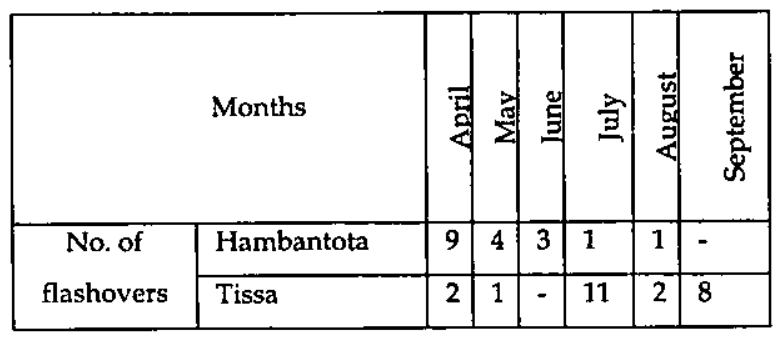

\section{Pollution Severity}

The performance of outdoor high-voltage insulators is adversely affected by environmental pollution in coastal and industrial regions. In some instances, solid pollution particles settle on the insulator surface and become a conductive electrolyte when the insulator surface is wetted by rain or fog. This allows leakage currents to flow over the insulator surface and lowers the electrical withstand voltage of the insulator, leading to possible flashover. IEC 815[2] describes on four levels of pollution such as Light, Medium, High and Very High. For each level of pollution, the corresponding minimum specific creepage distance for ceramic insulators as per IEC 815 is given in Table 2.

Table 2- IEC Recommended specific creepage distance over pollution severities.

\begin{tabular}{|c|c|}
\hline Pollution Level & $\begin{array}{c}\text { Minimum specific } \\
\text { creepage distance } \\
(\mathrm{mm} / \mathrm{kV})\end{array}$ \\
\hline I-Light & 16 \\
\hline II-Medium & 20 \\
\hline III-Heavy & 25 \\
\hline IV-Very \\
heavy
\end{tabular}

\section{Selection of pollution severity using ESDD method}

The measurement of ESDD in case of porcelain and glass has been standardized in the International Electro Technical Commission (IEC) document 60507 [1]. ESDD is measured by dissolving the contaminants on the surface of the insulators, in pure water and then measuring the conductivity of the water.

The solution conductivity is calculated using the following formula:

$$
\sigma_{20}=\sigma_{\theta}[1-b(\theta-20)]
$$

where,

$\sigma_{20}$ is the volume conductivity at a temperature of $20^{\circ} \mathrm{C}(\mathrm{S} / \mathrm{m})$

$\sigma_{\theta}$ is the volume conductivity at a temperature of $\theta^{\circ} \mathrm{C}(\mathrm{S} / \mathrm{m})$

$\theta$ is the solution temperature $\left({ }^{\circ} \mathrm{C}\right)$

$b$, is the factor depending on temperature $\mathrm{T}$

The salinity $\mathrm{S}\left(\mathrm{kg} / \mathrm{m}^{3}\right)$ of the solution is determined, when $\sigma_{20}$ is within the range of $0.004-0.4 \mathrm{~S} / \mathrm{m}$ by the use of following formula:

$$
S_{a}=\left(5.7 \sigma_{20}\right)^{1.03}
$$

The Equivalent salt deposit density ESDD $\left(\mathrm{mg} / \mathrm{cm}^{2}\right)$ is then obtained by the formula:

$$
E S D D=\frac{S_{a} \cdot V}{A}
$$

where, $\mathrm{S}_{\mathrm{a}}$ is the salinity $\left(\mathrm{kg} / \mathrm{m}^{3}\right)$

$\mathrm{V}$ is the volume of the solution $\left(\mathrm{cm}^{3}\right)$

$A$ is the area of the surface of Insulator $\left(\mathrm{cm}^{2}\right)$

\section{Selection of test sites}

Hambantota, a town in the south of Sri Lanka was selected as a sample area, since it is an area in which the electrical distribution system is affected by frequent insulator flashovers. Three zones have been selected as Zone 1 , Zone 2 and Zone 3 as per the distances shown in Table 3. Sample insulators were placed in respective zones as shown in Figure 1. 


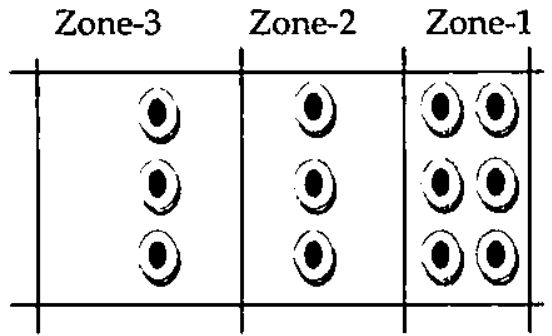

Figure 1- Sample insulator arrangements in three zones

Table 3- Pollution zones with distance from coastal boundary

\begin{tabular}{|c|l|c|}
\hline $\begin{array}{c}\text { Zone } \\
\text { No. }\end{array}$ & Zone Type & $\begin{array}{c}\text { Distance from coastal } \\
\text { boundary }(\mathrm{m})\end{array}$ \\
\hline 1 & Coastal & $0-300$ \\
\hline 2 & $\begin{array}{l}\text { Semi- } \\
\text { coastal }\end{array}$ & $300-1000$ \\
\hline 3 & Inland & $1000-3000$ \\
\hline
\end{tabular}

\section{Procedure and data collection}

Clean insulators, arranged as shown in Figure 1 were kept in service for a pre-determined period of one to six months under natural conditions. They were washed each month and the conductivity of the solutions was determined and is tabulated in Table 4 for all 3 zones.

Table 4- Conductivity Data for all zones

\begin{tabular}{|c|c|c|c|}
\hline \multirow{2}{*}{$\begin{array}{c}\text { Exposure } \\
\text { duration } \\
\text { (months) }\end{array}$} & \multicolumn{3}{|c|}{ Conductivity $(\mu \mathrm{s} / \mathrm{cm}$ ) } \\
\cline { 2 - 4 } & Zone-1 & Zone-2 & Zone-3 \\
\hline 1 & 669 & 547 & 526 \\
\hline 2 & 699 & 598 & 586 \\
\hline 3 & 1180 & 900 & 830 \\
\hline 4 & 2500 & N/A & N/A \\
\hline 5 & 2030 & N/A & N/A \\
\hline 6 & 2190 & N/A & N/A \\
\hline
\end{tabular}

N/A - Not available

By using Equations (1), (2) and (3), the ESDD values for each case was calculated, and is plotted against the exposure period to the environment as shown in Figure 2.

It is apparent from the Figure 2 that Zone- 1 ha sufficient data to forecast the future. It shows a significant variation of ESDD over the period of study. The Zones 2 and 3 having only 3 months data shows marginal variation of ESDD over the exposed period. The pattern of variation is seen to be similar with Zones 2 and 3 experiencing lower ESDD values as expected. Thus, only Zone-1 is further analyzed.

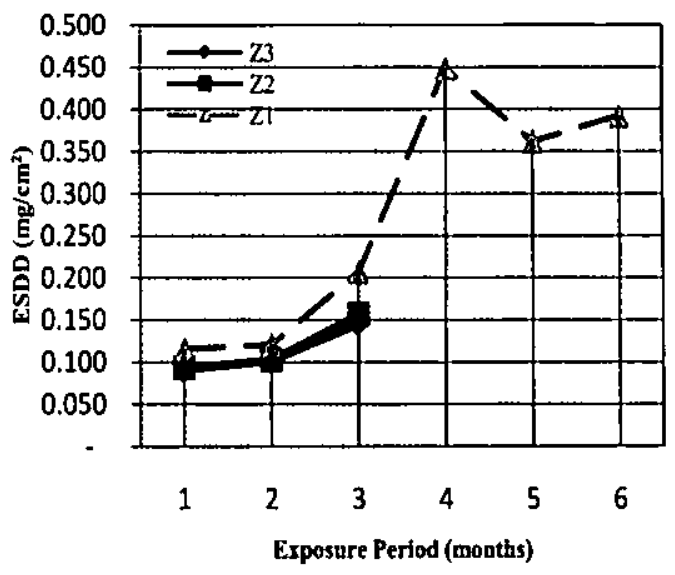

Figure 2- ESDD pattern variations

\section{Power frequency and Impulse test over artificial pollution}

To obtain a relationship between the ESDD value and the conductivity of the solution obtained from washing, sample insulators were hung individually in the high voltage laboratory after being artificially sprayed with salt of known concentration.

The contamination slurry to be applied on the insulator was obtained by mixing salt in one liter of water and spraying on top on the insulator. For this experiment, salt of five different weights were mixed with $1 \mathrm{~L}$ of water and sprayed on top of the each insulator, expecting to obtain a wide range of contamination levels represented by the ESDD. The five sample insulators applied with different salt levels, to represent the contamination, were subject to a power frequency test and thereafter for an impulse test. This process was repeated for 3 attempts and at each test data were recorded to calculate the average Flashover Voltage (FOV) values. In each attempt the insulators were damped with water spray thereby creating an artificial wet condition in the laboratory. The damping used was insufficient to remove the contamination from the insulator, but just sufficient to keep it moist.

\section{A. Power frequency test results}

Figure 3 shows the variation pattern of flashover voltage over the ESDD under the power frequency test. The Trend curve for the experimented results is assumed to be exponential. The curve has been extrapolated for more values of ESDD based on the tabulated results. 


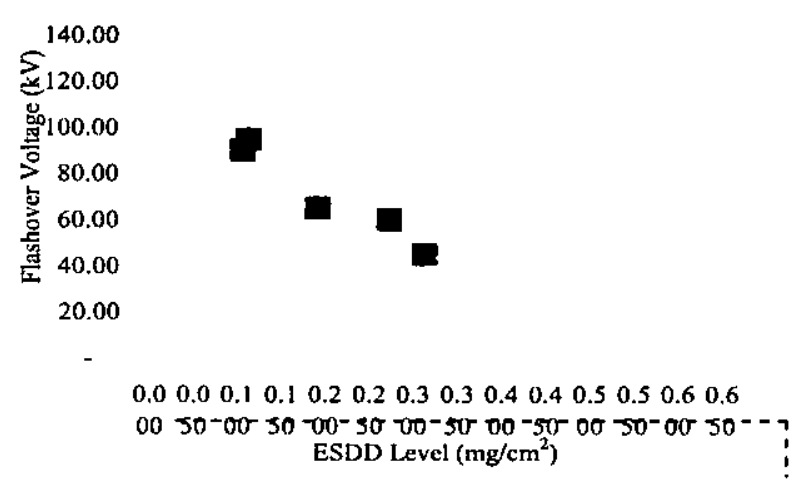

Figure 3- Flashover Voltage variations under power frequency test.

The operating voltage $V_{P E}$ (phase to Earth Voltage) in $33 \mathrm{kV}$ system is $19 \mathrm{kV}$. Based on Figure 3, for the insulator to flashover under the operating voltage of $19 \mathrm{kV}$, the ESDD value should reach a value of $0.6 \mathrm{mg} / \mathrm{cm}^{2}$.

Further, a non contaminated insulator was subjected for power frequency test and observed flash over voltage of $100 \mathrm{kV}$.

\section{B. Impulse Test results}

Figure 4 gives the variation pattern of flashover voltage over the ESDD under impulse test. Trend curve for the experimented results is assumed to be exponential.

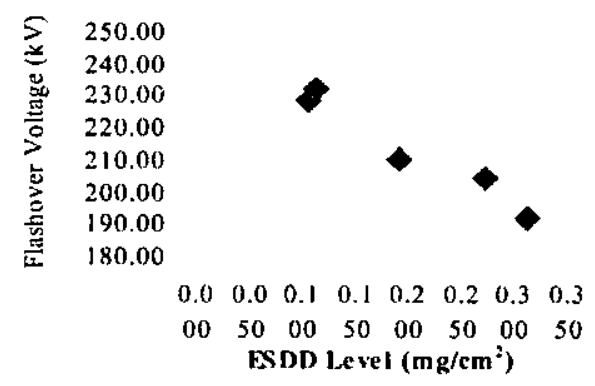

Figure 4-Flashover voltages under different ESDD values

Further, a non contaminated insulator was subjected for impulse test and observed flash over voltage of $240 \mathrm{kV}$.

\section{Prediction of Flashover Voltages under Power Frequency and Impulse Test}

Based on Figure 3 and 4, flashover voltages under the two tests are predicted by the trend curve and tabulated in table 5 for some ESDD values.

Table 5- Flashover Voltage Vs ESDD for power frequency and Impulse Test

\begin{tabular}{|c|c|c|}
\hline \multirow{2}{*}{$\begin{array}{c}\text { ESDD } \\
\left(\mathrm{mg} / \mathrm{cm}^{2}\right)\end{array}$} & \multicolumn{2}{|c|}{ Flashover Voltage (kV) } \\
\cline { 2 - 3 } & $\begin{array}{c}\text { Power Frequency } \\
\text { Test }\end{array}$ & $\begin{array}{c}\text { Impulse } \\
\text { Test }\end{array}$ \\
\hline 0.1 & 94.36 & 231.01 \\
\hline 0.2 & 68.38 & 212.61 \\
\hline 0.3 & 49.55 & 195.67 \\
\hline 0.4 & 35.91 & 180.09 \\
\hline 0.5 & 26.03 & 165.75 \\
\hline 0.6 & 18.86 & 152.54 \\
\hline
\end{tabular}

\section{Preventive maintenance}

In zones where there is pollution, besides having a good selection of the insulator, it is advisable to have a maintenance plan to treat the insulators. This is more important in areas with severe environment pollution or low rain probability, being necessary for the elimination of the pollutant layer placed on the insulator. This maintenance can be carried out with the system energized or de-energized.

Figure 2 is extrapolated for Zone- 1 so as to forecast a preventive maintenance procedure as shown in Figure 5.

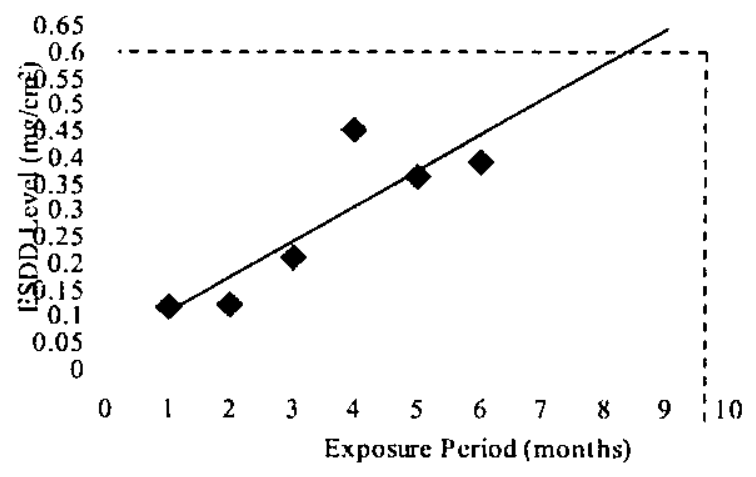

Figure 5- ESSD variation pattern over exposure duration for Zone-1

ESDD values for Zone -1 are plotted against the insulator exposure duration of a 6 months period. The actual trend for data points may not be exactly linear if the study is carried out over a longer period. Assuming a linear trend line, the curve is drawn. It cuts the $0.6 \mathrm{mg} / \mathrm{cm}^{2}$ ESDD value (related to the operating voltage of 
$19 \mathrm{kV}$ ) approximately at the $8^{\text {th }}$ month. This implies that insulators have to be cleaned artificially or naturally after 8 months from the date of fist cleaning as shown in Figure 5.

\section{Calculation of Specific Creepage Distance}

Creepage distance of the sample insulator is $900 \mathrm{~mm}$ whereas the maximum operating voltage $V_{L-L}$ (Line to Line Voltage) is $36 \mathrm{kV}$. Hence, the SCD for the $33 \mathrm{kV}$ pin insulator $(900 / 36)$ is $25 \mathrm{~mm} / \mathrm{kV}$. The analysis has been done using pin type insulators of $25 \mathrm{~mm} / \mathrm{kV}$ specific creepage distance and recommended pollution severity of the insulators to be 0.3 $0.6 \mathrm{mg} / \mathrm{cm}^{2}$ as per IEC 815 [2]. Figure 3 shows that the cut-off limit of pollution severity under operating voltage $(33 / \sqrt{3}=19 \mathrm{kV})$ is considered as $0.6 \mathrm{mg} / \mathrm{cm}^{2}$. This indicates that insulators have to be treated before reaching this pollution severity of $0.6 \mathrm{mg} / \mathrm{cm}^{2}$.

It is seen from the above analysis that there is no requirement to increase the specific creepage distance from $25 \mathrm{~mm} / \mathrm{kV}$ to the next upper level provided the insulators treatment is done according to the proposed procedure under preventive maintenance. This analysis describes one important fact that there is a possibility of using lower specific creepage distances such as 16 and $20 \mathrm{~mm} / \mathrm{kV}$ for inland sites where lower pollution or ESDD is obtained.

\section{Conclusions and Recommendations}

There is no streamlined procedure yet adopted for treating contaminated insulators under preventive maintenance. Under this study, an effort has been taken to introduce a certain mechanism to determine as to which period insulators should be treated and the types of remedial actions to be taken and the optimal solution. Severest pollution is experienced in the coastal area characterized by the $0-300 \mathrm{~m}$ range from the coastal boundary. At semicoastal and inland sites, the ESDD value is almost in an order of magnitude lower than that experienced in the coastal belt. Study implies that insulators in the Zone-1 have to be treated after 8 months from the date of the last washing under preventive maintenance.

The decrease of pollution will depend on the type of insulator, the maintenance, the increase of the creepage distance, a better design of the insulators etc. They are subjected to conditions that depend on the place at which they are installed. These conditions can vary extensively from one place to another, depending on the characteristics of the region considered. These characteristics make it possible for the level of insulation required to vary within the same line, due to the different conditions of the pollution at various points. The weather factors also influence in a very important way on the growth of the pollution levels in a region.

It is recommended to expose insulators for at least 2 years, without artificial washing, to tune up the results. In this regard, the $33 \mathrm{kV}$ pole line of $2 \mathrm{~km}$ length which runs in the coastal zone exposed to the sea breeze is suggested to be selected as a sample area. The CEB currently installs $33 \mathrm{kV}$ pin type insulators of $25 \mathrm{~mm} / \mathrm{kV}$. If insulators are treated as per the recommended schedule (before reaching 0.6 $\mathrm{mg} / \mathrm{cm}^{2}$ ESDD level), there is no need to increase the creepage distance to reduce insulator flashover. The CEB is also installing $25 \mathrm{~mm} / \mathrm{kV}$ insulators in the distribution network in inland sites irrespective of pollution severity. The requirement of the high specific creepage distance of $25 \mathrm{~mm} / \mathrm{kV}$ for inland sites, where the pollution severity is minimum, has to be reviewed through a comprehensive analysis which could be exercised exposing insulators with different specific creepage distances (16, 20 and $25 \mathrm{~mm} / \mathrm{kV}$ ) under real site condition for well over 2 years. If required level of specific creepage distance for inland sites could be reduced to 16 or 20 , a considerable saving can be achieved on insulators. It is also essential to study the antipollution performances of glass insulators over porcelain insulators with the same profile and for this a comprehensive study is needed to find the optimum solution having exposed both types of insulators under the same field condition for a reasonable period.

\section{References}

[1] IEC Publication 60507, "Artificial Pollution Tests on HV Insulators to be used on AC Systems", Second Edition, 1991.

[2] IEC Publication 60815, "Guide for the Selection of Insulators in respect of polluted Conditions", First Edition, 1986.

[3] F. Zedan, M. Akbar, A. Farag and H. Zarzoura, "Insulator Contamination Research in Saudi Arabia", March 1989.

[4] R. Wilkins, "Flashover voltage of high voltage insulators with uniform surface pollution films", Proceedings of the IEEE, 116, 457-465, 1969. 
[5] A. Rumeli and S. M.El-debeiky, "Pollution Flasover performance of High Voltage Insulators around Jeddah Region", Medical Journal of Islamic Academy of science 3:1, $1-5,1990$.

[6] P. Lambeth, H. Auxel and M. Verma, "Methods of Measuring the Severity of natural Pollution as it Affects HV Insulator Performance", Electra No.20, pp.37-52, 1972.

[7] Md.A. Salam, Z. Nadir, M. Akbar and Md.S Islam, "Study the Effects of Different types of Contaminants on the Insulator Resistance", Second International Conference on Electrical and Computer Engineering ICECE 2002, Dhaka, Bangladesh, 26-28 December 2002.

[8] IEC Publication 71-2, "Application Guide", Part 2, Third Edition, 1996.

[9] S. Ray, An Introduction to High Voltage Engineering, Pantnagar, India, 2004.

[10] W.H. Schwardt, J.P. Holtzhausen, W.L. Vosloo, "A comparison between measured Leakage Current and Surface Conductivity during Salt Fog Tests", Stellenbosch, South Africa.

\section{Acknowledgement}

This work has been carried out at the Department of Electrical Engineering, University of Moratuwa. Thanks are due to Head of the department and the technical staff of the high voltage laboratory, for permitting us to carry out the research programme. Thanks are also due to all our colleagues who helped us in numerous ways in making this project a success. 